\title{
Automating Ambulatory Practice Surveillance for Influenza-Like IIIness
}

\author{
Andrew Walsh* \\ Health Monitoring, Pittsburgh, PA, USA
}

\section{Objective}

To investigate the viability of using prediagnostic syndromic surveillance data from ambulatory practices for influenza-like illness surveillance

\section{Introduction}

Data submitted to ILINet from ambulatory practices are a primary feature of influenza-like illness (ILI) surveillance in the United States. Practices count relevant patient records and submit this data manually to ILINet. The ongoing data collection is useful for surveillance, and a significant amount of historical data has accumulated which is useful for research purposes and comparisons of the present season to the past. However, the tabulation of this data is costly, and retention of sentinel practices can be challenging as there is no mandate to submit data.

Increasingly, the EpiCenter syndromic surveillance system is receiving data from ambulatory practices. Syndromic surveillance data is sent automatically in near-realtime. Meaningful Use requirements incentivize practices to participate in ongoing data transmission. Syndromic surveillance data from ambulatory practices is thus a possible substitute for the current, more labor-intensive surveillance of ambulatory practices.

\section{Methods}

Chief complaints, triage notes and patient temperature were collected from 141 hospitals and 710 ambulatory practices for patients from 5 Philadelphia-area counties. An ILINet-compatible classification was defined using cough or sore throat in either the chief complaint or triage notes along with either mention of fever in one of those fields or a measured patient temperature over $100^{\circ} \mathrm{F}$. The existing EpiCenter classification of ILI Specified, which looks for reference to influenza or ILI in the text fields, was also used. Registrations were classified using both criteria.

\section{Results}

A total of 1,497,521 ED registrations and 836,431 ambulatory registrations were collected from June 30, 2014 to June 28, 2015. Of these, $10,661(0.71 \%)$ ED registrations and 2,359 $(0.28 \%)$ ambulatory registrations were classified as ILINet compatible, while $18,363(1.23 \%)$ ED registrations and 11,088 (1.33\%) ambulatory registrations were classified as ILI specified. Figure 1 shows the weekly time series of the percentage of visits by classification. The registrations classified as ILI specified more closely matched the expected values, with a baseline around $2 \%$ of visits and a peak of $4 \%$. The temporal pattern of these registrations was also most closely matched the expected pattern for flu season.

The percentage of ambulatory visits with an ILI specified increased earlier in flu season than the ED visits. This pattern was not consistent across all counties. Philadelphia County in particular exhibited minimal change in ILI visits at any point during flu season.

\section{Conclusions}

The current criteria used to define ILI-related visits for ILINet may not be applicable to patient-provided data. Although the ILINet criteria use symptoms that patients can identify and report, in practice those symptoms are not always individually recorded in chief complaints for ILI patients. A registrar is more likely to summarize multiple symptoms as "flu symptoms," "URI" (for upper respiratory infection), or similar. In essence, the registrar is performing a first level of classification into an ILI category. If syndromic surveillance data is to be used for ILI surveillance, the criteria for classifying visits will need to account for this summarizing of symptoms by the data providers.

The ILI time series suggest different usage patterns of ambulatory practices and EDs at different points in the flu season. This may be indicative of access to ambulatory care; patients may seek treatment there earlier if they can, while those without ambulatory access will wait longer until their disease is severe enough to warrant visiting the ED. This could explain the geographic differences observed, but it cannot explain all of the temporal differences, as influenza does not typically span a month or more even without treatment. It may also be the case that other, milder respiratory illnesses circulate in the fall.

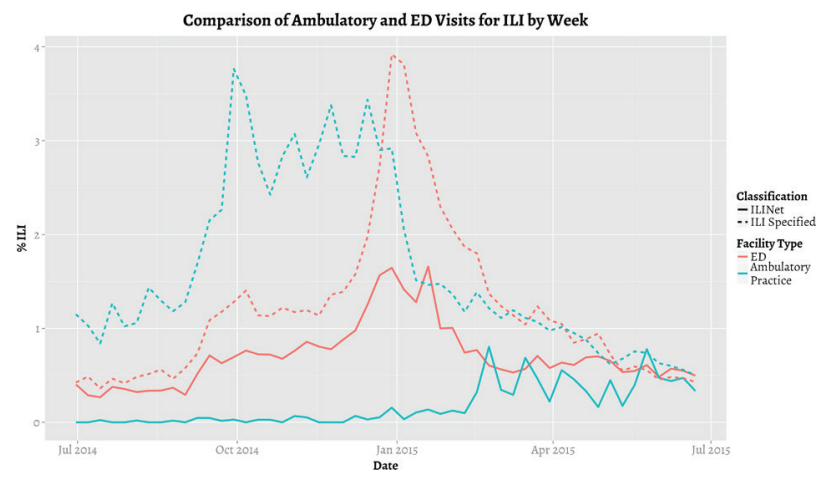

Figure 1: Weekly time series of the percentage of ILI-related healthcare visits to EDs (red) and ambulatory practices (blue) based on either standard ILINet symptoms (solid) or mention of influenza or ILI (dashed) in the prediagnostic text.

\section{Keywords}

ILINet; ambulatory; syndromic surveillance; influenza

\section{Acknowledgments}

We wish to thank the Pennsylvania Department of Health for funding support and data for this work.

\section{*Andrew Walsh}

E-mail: andy.walsh@hmsinc.com 